\title{
Pasta blanqueada y azúcares fermentables a partir de médula de bagazo de caña
}

\author{
Bleached pulp and fermentable sugars from sugarcane \\ pith bagasse
}

\author{
J. Jesús Vargas-Radilloł*, Edgar Salazar-Ríos', Lucia Barrientos-Ramírez', Armando Pérez-Centeno², Maite Rentería- \\ Urquiza³, Antonio Rodríguez-Rivas', Fernando Navarro-Arzate' y José Rutiaga-Quiñones ${ }^{4}$
} 'Departamento de Madera, Celulosa y Papel. CUCEI.
Universidad de Guadalajara. Jalisco, México.
*Autor de correspondencia. jvargasr@admcyp.cucei.
udg.mx

\author{
2 Departamento de Física, CUCEI. Universidad de Gua- \\ dalajara. Jalisco, México. \\ ${ }^{3}$ Departamento de Química, CUCEI-Universidad de
}

Guadalajara. Jalisco, México.

\section{RESUMEN}

La médula del bagazo de caña es un residuo subaprovechado que se produce en gran cantidad en los ingenios azucareros y en las fábricas de papel. Es una mezcla de células de parénquima, vasos y pequeñas fibras. Su contenido de carbohidratos y lignina es similar a la fracción fibra. Con el propósito de aprovechar este considerable contenido de carbohidratos, se aplicaron a la médula dos procesos distintos: Pulpeo-blanqueo, hidrólisis enzimática. El primer caso se aplicó prehidrólisis, pulpeo químico, blanqueo y refinación, obteniendo pulpa de médula blanqueada con $68 \%$ ISO de blancura y $0,38 \%$ de lignina residual. El mayor incremento en resistencia $(77,6 \%$ de aumento en índice de tensión, IT) fue con la incorporación de 30\% de pulpa de médula blanqueada sin refinar, mientras que con $10 \%$ de pulpa de médula blanqueada refinada, el IT se incrementó en 43,8\%. En el segundo caso, se trató enzimáticamente la médula. Con 6 h de hidrólisis se obtuvo $86,6 \%$ de rendimiento en azúcares fermentables tanto para pulpa de médula cocida como para pulpa de médula blanqueada, lo que representa cerca del $100 \%$ de hidrólisis de los carbohidratos presentes en el sustrato, lo cual es significativo ya que el rendimiento en azúcares fermentables de médula sin tratar fue de solo $26,1 \%$.

Palabras clave: blanqueo, material de compuesto, morfología, parénquima, sosa-AQ.

\section{ABSTRACT}

Pith bagasse is a sub-exploited residue produced in large quantities in sugar mills and paper mills. It is a mixture of parenchyma cells, vessels and small-size cellulose fibers. The content of carbohydrates and lignin in pith is similar to the fiber fraction. In order to take advantage of this significant carbohydrate content, two distinct processes were applied to the pith material: Pulping-bleaching, and enzymatic hydrolysis. In the first case the pith was treated with pre-hydrolysis, soda pulping, bleaching and beating process, obtaining bleached pith-pulp with $68 \%$ ISO brightness and $0,38 \%$ of residual lignin. The largest increase in strength $(77,6 \%$ increase in tensile index, IT) happened when $30 \%$ of this unbeaten pulp was added to paperboard, while with $10 \%$ of refined bleached pulp, IT increased in $43,8 \%$. In the second, the pith was enzymatically hydrolyzed. In 6 h of hydrolysis a yield of $86,6 \%$ of fermentable sugars was obtained in both, cooked pith-pulp and bleached pith-pulp, close to $100 \%$ hydrolysis of carbohydrates present in the substrate, which is significant because the yield of fermentable sugars from untreated pith was only $26,1 \%$.

KEY WORDs: bleaching, composite, morphology, parenchyma, soda-AQ.

\section{INTRODUCCIÓN}

La caña de azúcar (Saccharum officinarum L.) es una gramínea perenne, originaria de Asia, adaptada a la mayoría de climas tropicales y subtropicales (Freitas Andrade y Colodette, 2014), con gran rendimiento agrícola de $60 \mathrm{t} \mathrm{ha}^{-1}-100 \mathrm{t} \mathrm{ha}^{-1}$ (FAO. 2008). En México el rendimiento 
fue de $78,7 \mathrm{t} \mathrm{ha}^{-1}$ en el ciclo 2012/13, la más alta hasta entonces (DOF, 2014). De la periferia al centro se observa epidermis, corteza o cáscara, haces fibrovasculares y médula o tejido fundamental (Triana et al., 1990). La corteza tiene fibras lignocelulósicas en forma de haces fibrovasculares estrechamente empaquetados (Isaac et al., 2013), rígidas, muy lignificadas, alargadas y de pared gruesa, formando tejido esclerenquimatoso, adecuadas para la industria papelera (Triana et al., 1990).

La médula (colénquima, clorénquima, anular y parénquima) son células de almacenamiento para solutos y alimento para la planta (Hegbom, 1992), de forma irregular y anisotrópica paredes finas y muy porosas (Triana et al., 1990), de estructura frágil y fracturada (Chimenez et al., 2014). Predomina en el interior del tallo junto con haces fibrovasculares inmersos distribuidos espaciadamente, siendo estos haces más numerosos en la periferia, donde forman un anillo sólido, con pequeñas fibras celulósicas finas, de paredes delgadas (Trianaet al., 1990; Rasul et al., 1999), de menores dimensiones biométricas que la fibras de la corteza.

La caña de azúcar es prensada para extraer la sacarosa en los ingenios azucareros. Esto produce una gran cantidad de residuo, consistente en una mezcla de fibras, vasos y médula denominado bagazo integral (Boopathy, 2004). El bagazo de caña de azúcar promedio contiene $35 \%$ médula y el resto es la fracción fibrosa, preferida para la producción de papel (Rainey et al., 2012). Ha sido usado como combustible en las calderas del ingenio para generar electricidad, como materia prima para la industria de celulosa y papel y como reforzante de compuestos poliméricos (Cao et al., 2006). Además se ha utilizado bagazo para productos de alto valor agregado (químicos y metabolitos) (Pandey et al., 2000), nanocristales o nanofibras (Teixeira et al., 2011; Gilfillan et al., 2014), y etanol celulósico (Emsley, 2008).

Tanto en los ingenios como en las fábricas de papel la médula constituye un residuo orgánico no aprovechado y plantea un serio problema de eliminación de residuos. El desmedulado mejora el bagazo para la producción de pulpas, aumenta el rendimiento y mejora la blancura y las propiedades de resistencia del papel (Aguilar-Rivera, 2011). Una fábrica de papel que procesa $300 \mathrm{t} \mathrm{d}^{-1} \mathrm{de}$ bagazo genera $160 \mathrm{t} \mathrm{d}^{-1}$ de médula (Jain et al., 2011). En los ingenios azucareros la mayoría de la médula se quema en la caldera, junto con el resto del bagazo. Tiene baja eficiencia energética, ya que su poder calorífico es de 71,4 $\mathrm{kJ} \mathrm{kg}^{-1}\left(17,07 \mathrm{kcal} \mathrm{kg}^{-1}\right)$ (Díez et al., 2010). La eliminación de médula es benéfica para el almacenamiento del bagazo en fábrica puesto que reduce en $50 \%$ la contaminación por polvo libre, la humedad, contaminación por lixiviados, y la combustión espontánea (Rainey et al., 2013).

Casi todas las referencias consultadas sobre el aprovechamiento de la médula se refieren a la obtención de azúcares fermentables y etanol (García-Kirchner y Huitron, 1996; Hernández-Salas et al., 2009; Dasgupta et al., 2013). Se encontró una menor cantidad de menciones sobre el uso de la médula en el refuerzo de pulpa y polímeros (Rutiaga et al., 2002a; Lee y Mariatti, 2008). En México la caña de azúcar se utiliza casi exclusivamente para la producción de azúcar (refinada, morena, piloncillo) con 6,92 millones de toneladas en el ciclo 2012/13 así como 16,7 millones de litros de alcohol en 5 ingenios (DOF, 2014), obteniéndose además melaza $\left(40 \mathrm{~kg} \mathrm{t}^{-1}\right)$ y bagazo de caña como subproductos, así como otros aprovechamientos de menor importancia como compostas agrícolas, vinazas, ceras, fibra absorbente, etc. (Coveca, 2008).

La médula generada en México (35\% de los 12,5 millones de toneladas de bagazo) (Flores et al., 2008), podría convertirse en un fuente de biomasa importante considerando que tiene una composición química similar a la fracción de fibra, con 68\%-69\% holocelulosa $(20 \%$ $21 \%$ hemicelulosa y $48 \%-49 \%$ de alfa celulosa) y $21 \%$ $22 \%$ de lignina (Sanjuán et al., 2001). Por otro lado, los fabricantes de papel y cartón utilizan de forma frecuente fibra reciclada OCC (Old Corrugated Containers), como fuente de fibra barata (Rivera et al., 2008). El reciclar papel y cartón reduce la contaminación del agua en 3\% y la ambiental en 74\% (Misman et al., 2008), pero este proceso degrada el papel por lo que es necesario añadir celulosa "virgen" para compensar la degradación del papel 
(De la Madrid, 2009). En este contexto, la médula del bagazo de caña podría utilizarse para reforzar matrices celulósicas poliméricas.

\section{OBJETIVOS}

Debido a su disponibilidad, origen biológico, composición química, morfológica y sub-aprovechamiento, el objetivo de este trabajo fue evaluar la transformación de la médula de bagazo de caña en dos productos de mayor valor agregado: 1) pulpa de médula blanqueada para reforzar cartón OCC reciclado y 2) azúcares fermentables utilizando pretratamiento químico e hidrólisis con enzimas.

\section{MATERIALES Y MÉTODOS}

\section{Materiales}

El bagazo de caña utilizado fue recolectado de la zafra 2012/2013 del Ingenio azucarero José Ma. Martínez de Tala, Jalisco. El bagazo se secó al medio ambiente, a temperatura promedio de $23{ }^{\circ} \mathrm{C}$, el tiempo necesario para obtener una humedad final de aproximadamente $10 \%$. Posteriormente se separaron la fracción fibra y la fracción médula por frotamiento manual en seco utilizando una malla con poros de $0,8 \mathrm{~mm}$ de diámetro. La fracción médula se trató secuencialmente por medio de prehidrólisis ácida, pulpeo sosa-antraquinona, blanqueo parcialmente libre de cloro elemental (ECF) y refinación. También se utilizó cartón OCC reciclado, con 62,4 de número de kappa y $57^{\circ}$ SR (165 CSF) de grado de refinación. Se hizo una clasificación de fibras del cartón por el método Bauer McNett (Tappi T 233).

\section{Prehidrólisis}

Se aplicó una prehidrólisis ácida con $0,1 \% \mathrm{de}_{2} \mathrm{SO}_{4}$, relativo a la médula base seca, en suspensión acuosa al $10 \%$ de consistencia, $150{ }^{\circ} \mathrm{C}$ y 30 minutos, utilizando un reactor eléctrico giratorio. Se evaluó el rendimiento y número de kappa (T $236 \mathrm{~cm}$ - 85), según condiciones publicadas previamente (Kordsachia et al., 2004; Freitas y Colodette, 2014), ajustadas a la médula de bagazo de caña.

\section{Pulpeo}

Se utilizó el proceso químico sosa-antraquinona (Sosa$A Q)$. Se realizó primero una etapa exploratoria con las variables sosa (\%), tiempo de reacción (minutos) y temperatura $\left({ }^{\circ} \mathrm{C}\right)$, a $10 \%$ de consistencia y $0,1 \%$ de AQ respecto al material en base seca, y se evaluó el rendimiento y número de kappa. Como resultado de este estudio previo, la médula fue tratada aplicando las condiciones para obtener pasta grado blanqueable (número de kappa alrededor de 20$)$, es decir con $15 \%$ de sosa $\left(\operatorname{como~} \mathrm{Na}_{2} \mathrm{O}\right), 141^{\circ} \mathrm{C}, 55$ minutos y $0,1 \% \mathrm{AQ}$, en un reactor eléctrico giratorio. Se evaluó número de kappa, rendimiento y viscosidad CED ( $\mathrm{T}$ 230 om-89).

\section{Blanqueo ECF}

Se aplicó la secuencia dióxido de cloro-extracción alcalina-dióxido de cloro-extracción-dióxido de cloro, $\mathrm{D}_{0} \mathrm{ED}_{1} \mathrm{ED}_{2}$, utilizando condiciones publicadas previamente (Rutiaga et al., 2002b; Freitas y Colodette, 2014), ajustadas a la médula y evaluadas previo a cada etapa. Se utilizó como agente principal de blanqueo el dióxido de cloro ya que mejora la limpieza de la pulpa mientras preserva su viscosidad (Kordsachia et al., 2004). Las condiciones de blanqueo fueron $\mathrm{D}_{0}: 0,24$ de factor, 3,9\% $\mathrm{ClO}_{2}, 60 \mathrm{~min}$; $\mathrm{D}_{1}: 0,16$ de factor, $1,2 \% \mathrm{ClO}_{2}, 60$ minutos; $\mathrm{D}_{2}: 0,1$ de factor, $0,18 \% \mathrm{ClO}_{2}, 120$ minutos. En todas las etapas se aplicó una temperatura de $70{ }^{\circ} \mathrm{C}$ y $10 \%$ de consistencia. Se evaluó el rendimiento y número de kappa en cada etapa, así como la viscosidad al final del blanqueo.

\section{Refinación, formación de hojas y pruebas fisicomecánicas}

La pulpa de médula blanqueada fue refinada en el equipo de laboratorio Jokro (ISO, 1979). Este molino centrífugo tiene el mismo principio que los otros refinadores: tratamiento mecánico por rotor-estator. Consiste en una tina giratoria con seis cavidades en donde se colocan cilindros de paredes ranuradas (estator) que tienen un elemento cilíndrico libre ranurado (rotor) que muele o refina la pulpa. Trabaja con muestras pequeñas de 16 gramos a $6 \%$ consistencia y $150 \mathrm{~min}^{-1}$ (revoluciones por minuto). 
Los efectos de la refinación (aplastamiento, cepillado, corte, hidratación, fibrilación interna y externa) son determinantes para el entrelazamiento y consolidación del papel (Escoto, 2009). La refinación se hizo a diferentes tiempos: 5 y 10 minutos de forma progresiva, y 30 minutos, tiempo en el que visualmente se apreció que la muestra estaba molida o pulverizada, formada por microelementos.

A continuación se hicieron hojas de cartón OCC, solo y combinado con $10 \%$ y $30 \%$ de pulpa de médula blanqueada sin refinar y refinada. Las mezclas se trataron en un desintegrador por $10 \mathrm{~min}$ a $3000 \mathrm{~min}^{-1}$, y luego se mantuvieron 30 minutos en agitación en un homogeneizador a $1,57 \%$ de concentración. Se hicieron hojas de $60 \mathrm{~g}$ $\mathrm{m}^{-2}$ en un formador Tappi (T 205 om-88), midiendo su grado de refinación por medio de pruebas de drenado, la cual se usa para medir la intensidad de la refinación. Se expresa tanto en la escala Schopper Riegler, ${ }^{\circ} \mathrm{SR}$ (ISO, 1999) de 0-100, como en la escala Canadian Estándar Freeness (T-227) de 700-0. Por último, las hojas secas fueron acondicionadas para realizar las pruebas de resistencia y ópticas (T 402 om-93).

\section{Microscopía}

Se tomaron imágenes con microscopio óptico y microscopio electrónico.

\section{Microscopio óptico}

Muestras de pulpa de médula blanqueada y pulpa de médula blanqueada refinada se tiñeron con safranina para ser dispersadas sobre un portaobjeto y fijadas con polietilenglicol (Franklin, 1937; Ruzin, 1999). A continuación se tomaron imágenes con un microscopio compuesto marca Wild. Se evaluaron las características biométricas de los elementos anatómicos usando una escala certificada montada en el ocular del microscopio.

\section{Microscopio electrónico de barrido de emisión de campo (FE-SEM)}

Con el propósito de tomar micrografías de pulpa de médula y hojas formadas, la pulpa se secó utilizando un liofilizador marca Freeze Dryer $10 \mathrm{~N}$ mientras que las hojas se secaron en horno a $105{ }^{\circ} \mathrm{C}$ durante 30 minutos. Las muestras fueron posteriormente montadas sobre una película de carbón conductora de doble cara y metalizadas con una capa de oro de $4 \mathrm{~nm}$ con un sistema de erosión catódica marca Spi. Las muestras fueron observadas utilizando un microscopio de barrido de emisión de campo marca Tescan modelo Mira3, con un detector a 20 $\mathrm{kV}$ para captura de imágenes. Todas las imágenes fueron adquiridas en la modalidad de electrones secundarios.

\section{Azúcares fermentables}

Se obtuvieron azúcares fermentables a partir de médula del bagazo prehidrolizado, cocido y blanqueado, en cada caso, ya que estos procesos funcionan como pretratamiento o método de acondicionamiento de la médula, previo a la hidrólisis enzimática. Se empleó una mezcla enzimática comercial (Sigma-Aldrich) de celulasas (Celluclast 1,5 L), glucosidasas (Novozyme 188) y $\beta$-xilanasas. Los tratamientos fueron hechos en un incubador marca Lab-tech con una carga de $11 \mathrm{FPU} \mathrm{g}^{-1}$ de celulasas, $20 \mathrm{CBU} \mathrm{mL}^{-1}$ de celobiasas y $2,5 \%$ de xilanasas, a $2 \%(\mathrm{w} / \mathrm{w}), 50{ }^{\circ} \mathrm{C}, 140$ $\min ^{-1}$ y $\mathrm{pH}$ de 4,8 . Se añadieron $100 \mathrm{ppm}$ de cloranfenicol y 100 ppm de hidrocloruro de tetraciclina, antibióticos que previenen la contaminación microbiana. Se utilizaron frascos cerrados con tapón de hule. De esta forma, a cada frasco se añadió $1 \mathrm{~g}$ de la muestra, $50 \mathrm{~mL}$ de buffer (ácido cítrico/citrato de sodio), $270 \mu \mathrm{L}$ de Celluclast, $50 \mu \mathrm{L}$ de Novozyme, $25 \mathrm{mg}$ de xilanasa y los antibióticos. Se tomaron muestras a 1 h, 6 h y 24 h de hidrólisis. Al final de la hidrólisis, las muestras fueron enfriadas en baño de hielo, para detener la reacción, filtradas, y ensayadas para azúcares fermentables por el método del ácido 3,5 dinitrosalicilico, DNS (Miller, 1959), usando un espectrofotómetro UV-Vis, a $540 \mathrm{~nm}$ de longitud de onda. Para calcular la concentración de los azúcares fermentables se construyó una curva con 10 puntos de concentración de glucosa en el intervalo de $0,2 \mathrm{mg} \mathrm{mL}^{-1}$ a 2,0 $\mathrm{mg} \mathrm{mL}^{-1}$ (Ghose, 1987).

\section{Muestreo Estadístico}

Los tratamientos cocimiento/blanqueo y azúcares fermentables se hicieron por duplicado, mientras que en el caso 
de las pruebas de resistencia físico-mecánica se realizaron 8 repeticiones. Se calculó el promedio $(\bar{x})$ y desviación estándar (s) para cada prueba, utilizando estadística descriptiva convencional.

\section{Resultados}

\section{Pre-hidrólisis, pulpeo y blanqueo}

En relación con los tratamientos de acondicionamiento y deslignificación (pulpeo) de la médula, en el caso de la prehidrólisis ácida se obtuvo un rendimiento de 70,3\% ( $\mathrm{s}=$ 1,28\%), con número de kappa de 134 (s=3,1), mientras que en el pulpeo se obtuvo pasta con número de kappa promedio de 18 ( $\mathrm{s}=1,67$ ), rendimiento de 45,9\% ( $\mathrm{s}=$ 0,12\%), y viscosidad de 16,02 (s=2,46) cp. El material fue a continuación blanqueado (Tabla 1). Se obtuvo una blancura final de 68\% ISO (T 218 om-91) y viscosidad de 12,50 (s=0,97), grado de polimerización de 536,3.

Tabla 1. Resultados del blanqueo de médula de bagazo de caña.

\begin{tabular}{ccc}
\hline Etapa & Rendimiento (\%) & No. Kappa \\
\hline$D_{\mathrm{O}}$ & 100 & 16,5 \\
$\mathrm{E}$ & 96,0 & -- \\
$\mathrm{D}_{1}$ & 81,3 & 7,2 \\
$\mathrm{E}$ & 92,8 & -- \\
$\mathrm{D}_{2}$ & 75,6 & 0,9 \\
\hline
\end{tabular}

$\mathrm{D}_{\mathrm{O}}=$ Etapa inicial de Dióxido de cloro inicial, $\mathrm{D}_{1}=$ Primera etapa de dióxido de cloro, $\mathrm{D}_{2}=$ Segunda etapa de dióxido de cloro, $\mathrm{E}=$ Extracción alcalina

Blancura final de 68\% ISO; viscosidad de 12,50; grado de polimerización de 536,3.

\section{Morfología de la médula blanqueada y efecto de} la refinación

En la figura1 se presentan imágenes de la pulpa de médula blanqueada y pulpa de médula blanqueada refinada, con el propósito de apreciar visualmente el efecto de la refinación.

Los elementos predominantes son el parénquima y vasos (Fig. 1A), aunque también se aprecian fibras peque- ñas de pared delgada. Las fibras tienen $0,189 \mathrm{~mm}$ de longitud (L), ancho (D) de 3,19 $\mu \mathrm{m}$ y $0,89 \mu \mathrm{m}$ de espesor de pared (d). Estas fibras procedentes de los haces fibro-vasculares del parénquima central de la caña son significativamente de menores dimensiones que las fibras de la cáscara o corteza, las cuales tienen $\mathrm{L}=1,13 \mathrm{~mm}, \mathrm{D}=20$ $\mu \mathrm{m}$ y d $=12 \mu \mathrm{m}$ (Sanjuán et al., 2001). En relación con el resto de elementos, Agnihotri et al. (2010) hallaron que el parénquima del bagazo tiene una longitud de $0,327 \mathrm{~mm}$, con ancho de $0,053 \mu \mathrm{m}$, vasos con $\mathrm{L}$ entre $1,2 \mathrm{~mm}$ a 1,6 $\mathrm{mm}$.

\section{Hojas de cartón con pulpa de medula blanqueada y pruebas físico-mecánicas}

En la figura 2 se presentan imágenes adquiridas en el microscopio electrónico de las hojas de cartón reciclado y de las hojas de cartón reciclado reforzado con pulpa médula blanqueada refinada: Las figuras 2 a y 2 c son imágenes de una hoja de cartón OCC, las figuras $2 \mathrm{~b}$ y $2 \mathrm{~d}$ son imágenes de hojas de cartón reforzadas con pulpa de médula blanqueada refinada.

Un mayor aumento (2000x) permite observar con mayor detalle la interacción entre el cartón y la médula, como en la figura 3, en la que se muestra la imagen de cartón OCC cargado con médula blanqueada sin refinar.

En la tabla 2 se presentan las propiedades de drenado y resistencia de las hojas de cartón y cartón mezclado con pulpa de médula.

\section{Azúcares fermentables}

Los resultados de rendimiento en azúcares fermentables son presentados en la tabla 3.

\section{DISCUSIÓN}

Los valores de la prehidrólisis y cocimiento de la médula aquí obtenidos son similares a los de publicaciones previas en que se ha procesado bagazo de caña. Freitas y Colodette (2014) aplicaron una prehidrólisis a $180^{\circ} \mathrm{C}$ y 15 minutos a bagazo desmedulado y obtuvieron $71,0 \%$ de rendimiento, atribuyendo la pérdida en biomasa principalmente a la solubilización de xilanas, mientras que 

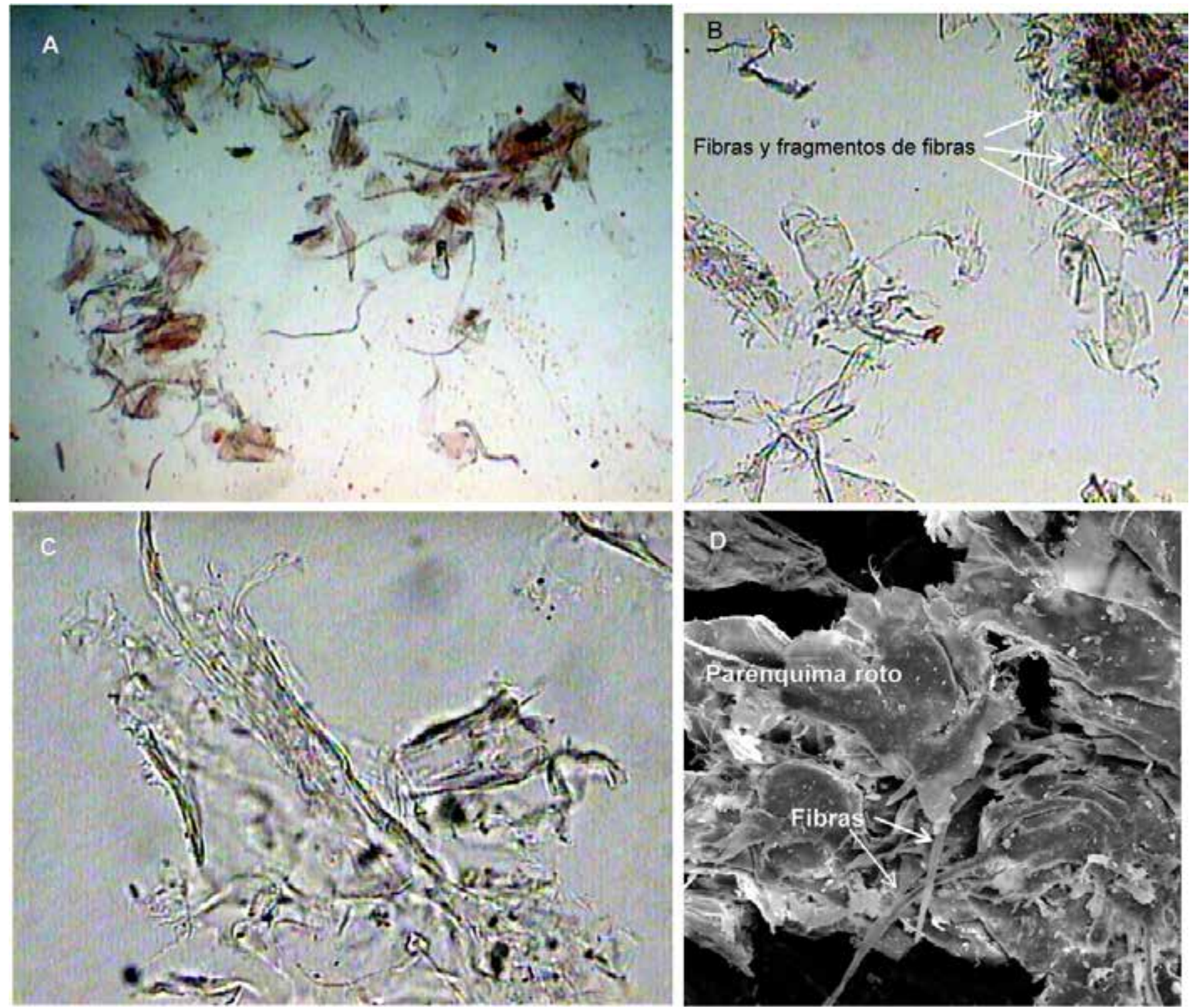

Figura 1. Imágenes de pulpa de médula de bagazo de caña: A. Pulpa de médula blanqueada, microscopio óptico, 4x; B. Pulpa de médula blanqueada refinada, microscopio óptico, 4x; C. Pulpa de médula blanqueada refinada, microscopio óptico, 40x; Pulpa de médula blanqueada refinada, microscopio electrónico, 500x.

Agnihotri et al. (2010) procesaron bagazo de caña desmedulado con una carga de $14,0 \%$ de álcali, $150{ }^{\circ} \mathrm{C}, 60$ minutos y $0,1 \%$ de $A Q$ obteniendo rendimiento de $42,2 \%$ y número de kappa de 16,1.

En el caso del blanqueo de la médula, aunque la blancura final no fue la óptima (68\% ISO), se eliminó gran parte de la lignina $(0,9 \%$ x $0,15 \%=0,13 \%$ de lignina residual teórica) (T $236 \mathrm{~cm}-85)$, mientras que la viscosidad disminuyó levemente en 3,52 cp. Estos resultados indican que el material procesado contiene elementos poliméricos factibles de transformarse en otros productos útiles.

En relación con la refinación, se conoce que este tratamiento produce fibrilación externa por desprendimiento gradual de las capas de la pared celular ( $\mathrm{P}_{\mathrm{y}} \mathrm{S}_{1}$ ), exposición de la pared $\mathrm{S}_{2}$ con fibrilación interna por pérdida de la pared celular (Wagberg, 2005). La fibrilación incre- 

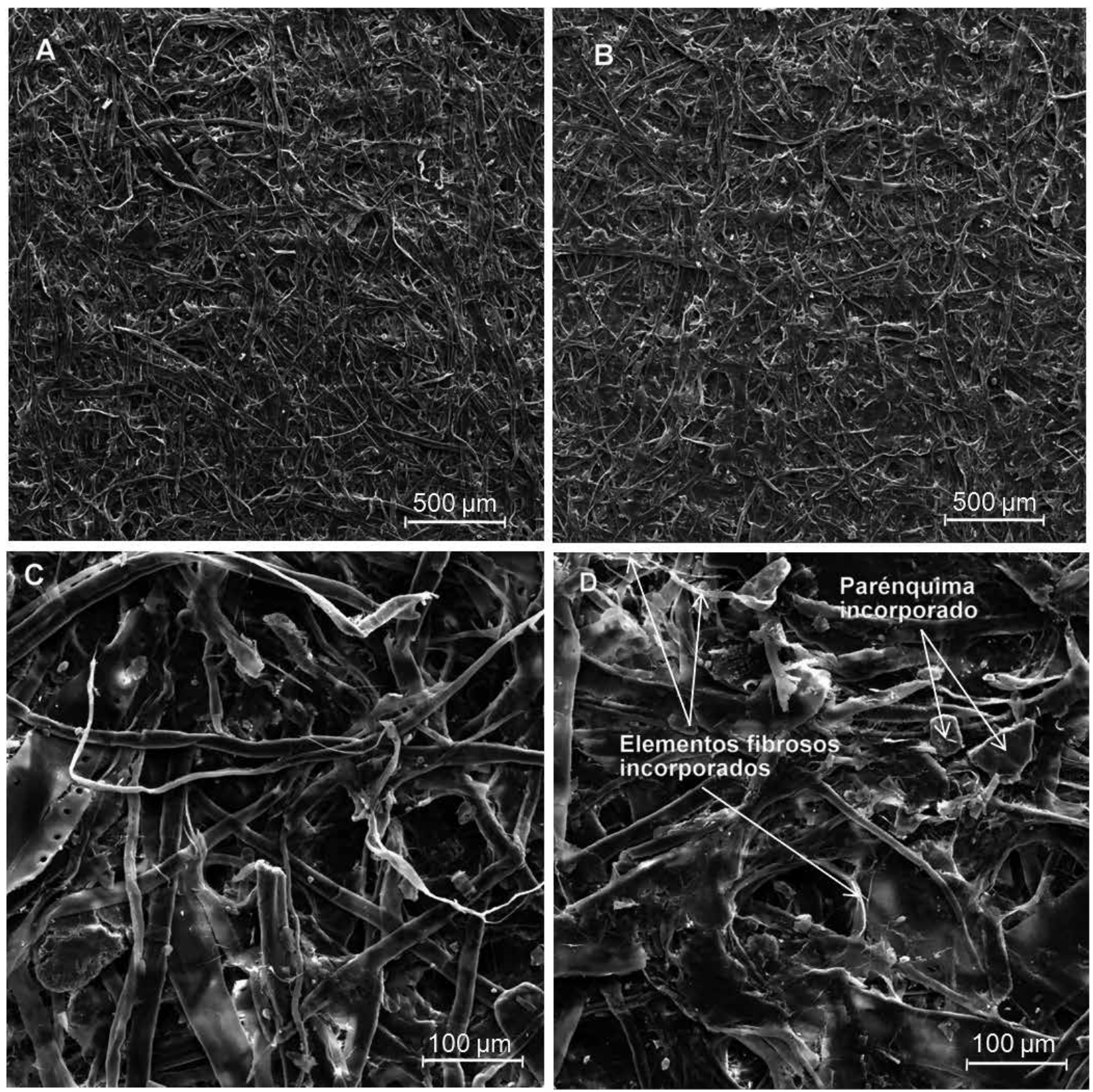

Figura 2. Imágenes de hojas de cartón solo y cargado con pulpa de médula blanqueada refinada: A. Cartón OCC, 100x; B. Cartón OCC con médula blanqueada refinada, 100x; C. Cartón OCC, 500x; D. Cartón OCC con médula blanqueada refinada, 500x.

menta las propiedades de resistencia mecánica de los compuestos (Nakagaito y Yano, 2005).

En este este caso, debido a que la médula blanqueada está formada por una mezcla de sus elementos anatómicos originales, y por tanto con alto contenido de parénquima y poco contenido fibroso, la refinación provocó rotura de las pequeñas fibras provenientes de los haces fibrovasculares de la parte central de la caña (Fig. 1C), lo que generó fibrillas o macrofibrillas (Fig. 1B), con probables diámetros entre $8 \mathrm{~nm}$ y $60 \mathrm{~nm}$ y varias micras de longitud (Fengel y Weneger, 1984) y finos, además de aplastamiento y rompimiento de la mayor parte del parénquima en fragmentos de diversos tamaños desde paredes celulares completas hasta fragmentos de pared celular (Fig. 1D).

Al incorporar la médula blanqueada al cartón OCC se producen efectos diversos. Las imágenes de microscopio 
Tabla 2. Propiedades de resistencia y drenabilidad del cartón, y del cartón reforzado con médula de bagazo de caña.

\begin{tabular}{|c|c|c|c|c|c|c|c|c|}
\hline Muestra & $\begin{array}{l}{ }^{\circ} \mathrm{SR} \\
(\mathrm{mL})\end{array}$ & $\begin{array}{l}\text { Drenado en } \\
\text { el formador } \\
\text { (segundos) }\end{array}$ & $\begin{array}{c}\text { Densidad } \\
\text { aparente } \\
\left(\mathrm{g} \mathrm{cm}^{-3}\right)\end{array}$ & $\begin{array}{c}\text { Índice de } \\
\text { tensión } \\
\left(\mathrm{N}-\mathrm{m} \mathrm{g}^{-1}\right)\end{array}$ & $\begin{array}{c}\text { Dobles } \\
\text { (No.) }\end{array}$ & $\begin{array}{l}\text { Índice de } \\
\text { explosión } \\
\left(\mathrm{kPa}-\mathrm{m}^{2} \mathrm{~g}^{-1}\right)\end{array}$ & $\begin{array}{c}\text { Índice de } \\
\text { rasgado } \\
\left(\mathrm{mN}-\mathrm{m}^{2} \mathrm{~g}^{-1}\right)\end{array}$ & $\begin{array}{l}\text { Porosidad } \\
\text { (segundos) }\end{array}$ \\
\hline \multicolumn{9}{|c|}{$\bar{x} \pm s$} \\
\hline C & 56 & $6,0 \pm 0,1$ & $0,39 \pm 0,02$ & $21,51 \pm 3,5$ & $7,7 \pm 2,4$ & $1,7 \pm 0,1$ & $6,21 \pm 0,0$ & $5,5 \pm 0,1$ \\
\hline С1OMB & 57 & $6,7 \pm 0,2$ & $0,40 \pm 0,01$ & $21,86 \pm 1,1$ & $4,7 \pm 1,5$ & $1,38 \pm 0,2$ & $6,70 \pm 0,4$ & $5,4 \pm 0,5$ \\
\hline C10MB5 & 65 & $10,2 \pm 1,2$ & $0,43 \pm 0,02$ & $28,86 \pm 2.4$ & $11 \pm 2,8$ & $1,92 \pm 0,1$ & $5,69 \pm 0,7$ & $15,6 \pm 3,5$ \\
\hline ClOMB1O & 66 & $9,9 \pm 1,1$ & $0,42 \pm 0,01$ & $29.83 \pm 1,1$ & $9,5 \pm 2,3$ & $2,16 \pm 0,1$ & $6,41 \pm 0,3$ & $13,2 \pm 1,2$ \\
\hline С10МB30 & 73 & $10,8 \pm 0,3$ & $0,42 \pm 0,02$ & $30,94 \pm 1,0$ & $12 \pm 2,4$ & $1,92 \pm 0,2$ & $5,47 \pm 0,0$ & $19,7 \pm 8,1$ \\
\hline СзОМВЗО & 90 & $66,2 \pm 8,3$ & $0,50 \pm 0,02$ & $27,09 \pm 2,3$ & $12 \pm 3,7$ & $2,07 \pm 0,3$ & $5,33 \pm 1,7$ & $342,0 \pm 15,5$ \\
\hline сзомВ & 66 & $9,5 \pm 0,4$ & $0,44 \pm 0,02$ & $38,20 \pm 3,9$ & $5,0 \pm 1,0$ & $1,31 \pm 0,2$ & $5,31 \pm 0,6$ & $31,0 \pm 0,7$ \\
\hline сзомс & 63 & $14,4 \pm 0,7$ & $0,45 \pm 0,01$ & $22,33 \pm 4,8$ & $4,5 \pm 0,5$ & $1,27 \pm 0,2$ & $5,20 \pm 0,4$ & $25,0 \pm 4,2$ \\
\hline
\end{tabular}

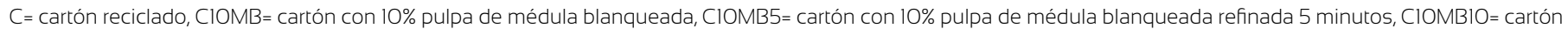
con 10\% de pulpa de médula blanqueada refinada 10 minutos, C1OMB30= cartón con 10\% de pulpa de médula blanqueada refinada 30 minutos, C30MB= cartón con 30\% de pulpa de médula blanqueada, C30MC= cartón con 30\% de médula cocida.

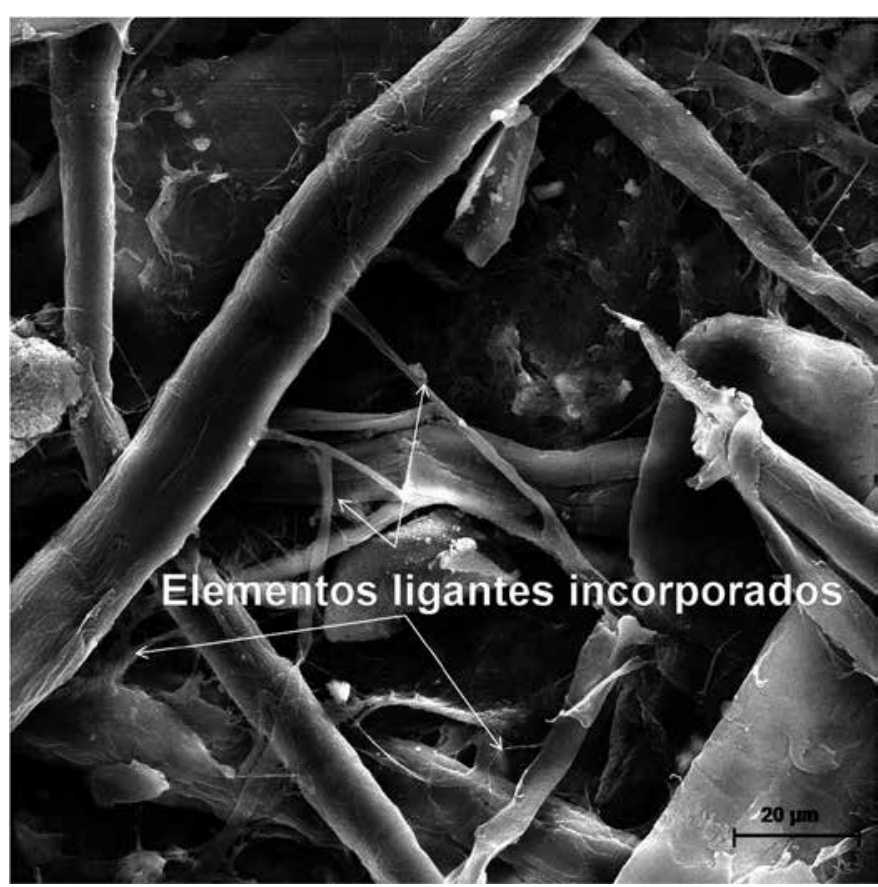

FIGURA 3. Imagen FE-SEM 2000x de hoja de cartón OCC con médula blanqueada añadida.

muestran que la hoja de cartón con médula (Fig. 2B) presenta menos huecos y menos rugosidad que la hoja de cartón sola (Fig. 2A). En la figura 2C (hoja de cartón a 500x), se pueden observar fibras con puntuaciones intertraqueidas areoladas propias de las fibras de pino, componentes
Tabla 3. Rendimiento de azúcares fermentables de la médula de bagazo de caña

\begin{tabular}{ccc}
\hline \multirow{2}{*}{ Muestra } & $\begin{array}{c}\text { Tiempo de } \\
\text { hidrólisis, } \mathrm{h}\end{array}$ & $\begin{array}{c}\text { Azúcares fermentables, \% } \\
\bar{x} \pm s\end{array}$ \\
\hline \multirow{3}{*}{ Médula cruda } & 1 & $8,03 \pm 1,3$ \\
& 6 & $8,03 \pm 0,5$ \\
& 24 & $26,10 \pm 1,2$ \\
\hline \multirow{3}{*}{ Prehidrolizada } & 1 & $22,20 \pm 1,1$ \\
& 6 & $43,94 \pm 0,4$ \\
\hline \multirow{2}{*}{ Cocida } & 24 & $49,12 \pm 2,7$ \\
& 1 & $79,74 \pm 1,1$ \\
& 24 & $86,60 \pm 0,6$ \\
Blanqueada & 1 & $31,08 \pm 2,1$ \\
\hline & 24 & $84,79 \pm 1,1$ \\
& & $36,60 \pm 1,2$ \\
\hline
\end{tabular}

del cartón OCC, así como finos ocasionados por el reciclaje. En la figura 2D (aumento 500x) se muestra el parénquima roto depositado en los huecos del cartón OCC, como relleno, así como fibras y fibrillas incorporadas desde la médula blanqueada, que interactúan con las fibras del car- 
tón. Asimismo en la figura 3 se aprecia que el parénquima blanqueado y las fibras pequeñas blanqueadas parecen interactuar con las fibras de cartón, en forma de ligaduras.

Respecto a las propiedades de resistencia (Tabla 2), la única que evidentemente mejoró fue la resistencia a la tensión (T 404 cm-92). Esta aumentó consistentemente cuando se agregó $10 \%$ de pulpa de médula blanqueada refinada 5 minutos (C10MB5) y refinada 30 minutos (C10MB30). En este último caso, se advirtió un incremento en IT, respecto al cartón OCC (C en la tabla), de 43,8\% (de $21,51 \mathrm{~N} \mathrm{~m} \mathrm{~g}^{-1}$ a $30,94 \mathrm{~N} \mathrm{~m} \mathrm{~g}^{-1}$ ), sin incremento significativo en el tiempo de drenado en el formador de hojas (equivalente al drenado en la mesa plana de la máquina de papel), de 6 segundos en el cartón OCC a 10,8 segundos del cartón reforzado con médula, ni aumento relevante de la porosidad Gurley (T $460 \mathrm{om}-88$ ), que indica la permeabilidad del papel con base en su estructura abierta o cerrada, de 5,5 segundos a19,7 segundos.

Sin embargo, añadir un exceso de médula blanqueada y refinada ocasiona una disminución en la resistencia, lo que se evidencia al comparar las muestras C10MB30 y C30MB30: con 30\% de pulpa de médula refinada 30 minutos (C30MB30), el índice de tensión (IT) cayó 12,4\%, de 30,94 N $\mathrm{m} \mathrm{g}^{-1}$ del cartón con $10 \%$ de médula a $27,09 \mathrm{~N} \mathrm{~m} \mathrm{~g}^{-1}$ del cartón con $30 \%$ de médula blanqueada refinada, ya que se incrementó la cantidad de parénquima roto añadido, el cual aparentemente no mejora las propiedades de resistencia. Además el tiempo de formación de hojas se incrementó considerablemente a 66,2 segundos (tiempo de formación de la muestra С30МB30), lo que indica un drenado sumamente lento, lo que afectaría la velocidad de producción a través del proceso de la máquina de papel (runnability), así como también una disminución significativa en la porosidad (porosidad de 342 segundos), lo que evidencia un papel cerrado. Esta disminución en drenado y en porosidad, se atribuye al parénquima roto que se deposita en los huecos del cartón OCC, como relleno, así como finos, los cuales reducen el tiempo de drenado (Paavilainen, 1993).

El mayor aumento en resistencia a la tensión, de 21,51 $\mathrm{N} \mathrm{m} \mathrm{g}^{-1}$ a $38,2 \mathrm{~N} \mathrm{~m} \mathrm{~g}^{-1}$, ganancia de $77,6 \%$ respecto al cartón oCC, lo ocasiona añadir 30\% de medula blanqueada sin refinar (С30MB). Este valor de resistencia se acerca al del papel de escritura producido a partir de fibra virgen, el cual tiene un valor entre $40 \mathrm{~N} \mathrm{~m} \mathrm{~g}^{-1}$ y $45 \mathrm{~N} \mathrm{~m} \mathrm{~g}^{-1}$ de IT (González et al., 2012). Un efecto distinto ocurrió al añadir 30\% de pulpa de médula cocida sin blanquear (C30MC), como se aprecia en la tabla 2, ya que no se mejoró ninguna de las propiedades de resistencia del cartón, lo que muestra lo valioso del blanqueo químico. La resistencia a la tensión o estiramiento se debe tanto a la resistencia individual de las fibras como a la resistencia de los enlaces interfibrilares (Page, 1969; González et al., 2012). El incremento de la tensión del cartón reforzado con pulpa de médula blanqueada se atribuye a las fibras y fibrillas (producidas con el blanqueo) contenidas en la médula de bagazo añadido, como se observa en las figuras presentadas arriba, así como a la incorporación de fibras enteras flexibles (Fig. 3), libres de lignina, hábiles para establecer enlaces intra e inter-fibrilares, además de parénquima sin refinar.

Adicionalmente, el blanqueo químico produce una desorganización de la morfología de fibras y médula y aumenta la rugosidad de su superficie, además de que se producen algunos microelementos (Chimenez et al., 2014). En estudios previos, Rutiaga et al. (2002a), mencionan que la pulpa de médula blanca, debido a su compatibilidad con el papel, actuaría como una "malla" entrelazante con las fibras. En esta mezcla (С $30 \mathrm{MB}$ con cartón OCC) la pulpa de médula blanqueada no ocasiona aumento importante en el tiempo de drenado (9,5 segundos) ni pérdida de porosidad (31 segundos). Se podría especular que la porosidad no se incrementa debido a que el parénquima añadido con la pulpa de médula blanqueada incorporada al cartón es parénquima entero, y al secarse el papel, este elemento se contrae, además que tiene menos finos que el material refinado. Asimismo, aumenta 10 unidades (de 56 a 66) el valor SR, ya que este material (parénquima) tiene elevada capacidad de absorción de líquidos y tamaño irregular lo que afecta la drenado (Triana et al., 1990).

Respecto al resto de propiedades, en todos los casos la resistencia a la explosión (T 403 om-91) no cambió o disminuyó levemente, mientras que la resistencia al rasgado (T 414 om-88) disminuyó un poco. Ya que la resis- 
tencia al rasgado depende en gran medida de la longitud de las fibras (Clark, 1969), añadir pulpa de médula blanqueada al cartón, lo cual implica menos fibra larga y más fibra corta y finos, provoca decremento de esta propiedad. Asimismo, la resistencia al doblez ( $\mathrm{T}$ 423) mejoró marginalmente.

La densidad aparente se incrementa con la adición de médula blanqueada y médula blanqueada refinada. Cartón reforzado con $30 \%$ de médula blanca refinada 30 minutos (С30MB30) presenta la mayor densidad aparente con $0,5 \mathrm{~g} \mathrm{~cm}^{-3}$, mientras que el cartón reciclado tiene el menor valor con $0,39 \mathrm{~g} \mathrm{~cm}^{-3}$. La densidad aparente del papel es una propiedad representativa de las características de la pasta celulósica, ya que es producto de múltiples factores (fibrilación, cantidad de fibrillas y microfibrillas, flexibilidad de las fibras, propiedades físicas de las fibras, cantidad de enlaces, etc.) (Amiri et al., 1991; Paavilainen, 1993; Gorres et al., 1996).

Es también destacable el elevado valor SR del cartón (C en la tabla 2 ), de $56^{\circ} \mathrm{SR}$ (170 CSF), característico de los cartones reciclados varias veces en un sistema cerrado (Howard y Bichard, 1992). El contenido alto de finos acumulados (38,3\% en este caso), no retenidos por la malla 200 , son los que mayor efecto negativo provocan en la resistencia del papel, entre otros factores, y además tienen alto grado de hinchamiento y absorción de agua (Htun y De Ruvo, 1978).

Existe un considerable número de referencias del uso de celulosa altamente refinada (celulosa microfibrilada) proveniente de materias primas fibrosas como refuerzo de papel. Por ejemplo, González et al. (2012) añadieron microfibras de eucalipto a pulpa blanqueada de eucalipto sin refinar, encontrando que con $9 \%$ de nanofibras el índice de tensión mejoró $100 \%$, el índice de explosión mejoró 2,72 veces, y el rasgado se incrementó $80 \%$, pero ocasionó un pobre nivel de drenado y porosidad Gurley, la cual aumento de 2 segundos a 52 segundos.

Son pocas las referencias respecto a la utilización de la médula del bagazo de caña blanqueada como refuerzo. Rutiaga et al. (2002a) añadieron pulpa de médula de bagazo de caña blanqueada a pulpa de pino blanqueada refinada a diversos niveles. Encontraron que el largo de ruptura se incrementó hasta 5 veces al añadir $10 \%$ de pulpa de médula blanqueada al pino refinado $12{ }^{\circ} \mathrm{SR}$, y hasta 7 veces al añadir $40 \%$ de ésta médula. Mientras que el índice de rasgado (IR) se incrementó 1,15 veces al agregar $10 \%$ de este material, disminuyendo este incremento al añadir mayor cantidad. Estos resultados son mejores que los aquí encontrados, posiblemente porque el sustrato utilizado fue diferente, en este caso cartón OCC, con fibras más rígidas y gran cantidad de finos. El papel reciclado, como es el caso del cartón occ, tiene pobre calidad y baja resistencia debido a la reducción de flexibilidad y capacidad de hinchamiento de las fibras a causa del fenómeno de hornificación ${ }^{1}$ (Scallan y Tydeman, 1992), y por la desactivación de agentes enlazantes de su superficie. La hornificación hace las fibras más rígidas, debido a que sufren un colapso interno a causa del proceso de secado, lo que dificulta su fibrilación (Minor et al., 1993).

Por otro lado, en relación con la producción de azúcares fermentables, el mejor rendimiento se obtuvo con la pulpa de médula cocida y la pulpa de médula blanqueada utilizadas como sustrato de hidrólisis, las cuales tuvieron un comportamiento similar: $86,6 \%$ de rendimiento en azúcares fermentables con $6 \mathrm{~h}$ de tratamiento, lo que significa que se hidroliza la mayor parte del $97,3 \%$ de carbohidratos de la pulpa de médula cocida, y la mayor parte del 99,9\% de carbohidratos de la pulpa de médula blanqueada. En ambos casos, un tiempo mayor de hidrólisis, $24 \mathrm{~h}$, provoca que el rendimiento de azúcares fermentables disminuya. Como era de esperarse, la médula con mayor contenido de lignina residual produce menor rendimiento: Con la médula cruda (sin ningún tratamiento) se obtiene un máximo de $26,10 \%$ de rendimiento, mientras que con la médula prehidrolizada se obtuvo un máximo en rendimiento de 49,12\%, ambos con 24 h de hidrólisis. En el caso de la pulpa de médula cocida y pulpa de médula blanqueada, el tratamiento químico eliminó la mayor parte de la lignina y disminuyó la cristalinidad de la celu-

\footnotetext{
1 Los cambios que ocurren en la fibra durante el proceso de secado corresponden a un fenómeno investigado en fibra virgen por Jayme (1944), al que se denominó Hornificación.
} 
losa, principales barreras que sirven de protección contra la actividad enzimática sobre los azúcares (Zheng et al., 2009). También se menciona que el blanqueo de la biomasa lignocelulósica hace más accesibles el material a la hidrólisis (Novaes et al., 2012). García-Kirchner y Huitron (1996), obtuvieron un máximo de 25,7\% de azúcares fermentables en 48 horas, al hidrolizar médula de bagazo de caña sin pretratamiento, utilizando una mezcla enzimática de endo y exoglucanasas, glucosidasas y xilanasas, cultivadas a partir de los hongos Penicillium sp y A. terreus Lo que coincide con el $27,0 \%$ de rendimiento obtenido con la hidrólisis enzimática por 24 h de la médula sin tratar (Tabla 3). En estudios más recientes (HernándezSalas et al., 2009), pretrataron la médula de bagazo de caña con $\mathrm{NaOH}$ diluido $(2 \% \mathrm{w} / \mathrm{v})$ a $121{ }^{\circ} \mathrm{C} ; 1,1 \mathrm{~kg} \mathrm{~cm}^{-2}$ y $4 \mathrm{~h}$, e hidrólisis posterior con $20 \%(\mathrm{w} / \mathrm{w})$ de una mezcla enzimática de Celluclast, Novozyme, Cellubrix y Pulpzyme HC, y obtuvieron solo $11 \%-20 \%$ de sacarificación. Dasgupta et al. (2013), utilizaron médula de bagazo pre tratada con vapor y $\mathrm{H}_{2} \mathrm{SO}_{4}$ e hidrólisis enzimática para obtener $40 \mathrm{~g} \mathrm{~L}^{-1}$ de azúcares fermentables y $17,4 \mathrm{~g} \mathrm{~L}^{-1}$ de alcohol.

\section{CONCLUSIONES}

Se evaluó el efecto de añadir médula de bagazo de caña como refuerzo de cartón OCC, llegando a las siguientes conclusiones:

La médula blanqueada promueve la interacción entre las fibras del cartón por medio de enlaces y mayor área de contacto a través de las células de parénquima blanqueadas, con aumento de la resistencia a la tensión.

Este material blanqueado, sin refinar, se puede añadir en altos porcentajes sin decremento de las propiedades de drenado y porosidad.

El batido (refinado) de la médula blanqueada provoca rompimiento del parénquima en fragmentos de diversos tamaños y finos no funcionales.

Los fragmentos y finos no funcionales actúan como relleno de huecos de las hojas, mejorando su rugosidad y opacidad, pero no la resistencia, porosidad y capacidad de drenado, si se añade en un porcentaje mayor a $10 \%$.
Probablemente otros métodos de refinación más suaves (Pila Valley o Pila Holandesa) promoverían una mayor generación de fibrillas o finos funcionales entrelazantes.

Desde otra perspectiva, la médula del bagazo de caña podría ser un material útil para transformarse en bioproductos, ya que la baja cristalinidad y grado de polimerización facilitaría la generación de azúcares fermentables.

\section{RECONOCIMIENTOS}

Deseamos expresar nuestro agradecimiento a la SEP-PROMEP por el apoyo económico del proyecto clave PROMEP/103.5/13/6982 “Obtención de azúcares fermentables y celulosa microfibrilada a partir de médula de bagazo de caña”, del cual se generó este manuscrito.

\section{REFERENCIAS}

Aguilar-Rivera, N. 2011. Efecto del almacenamiento de bagazo de caña en las propiedades físicas de celulosa grado papel. Ingeniería Investigación y Tecnología 12(1):189-197.

Agnihotri, S., D. Dutt y C.H. Tyagi. 2010. Complete characterization of bagasse of early specie of Saccharum officinarum-co89003 for pulp and papermaking. BioResources 5(2):1197-1214.

Amiri, R., J.R.Wood, A. Karnis y J. Gorres. 1991. The apparent density of paper. Proc. International Paper Physic Conference. p:11-18.

Boopathy, R. 2004. Use of post-harvest sugarcane residue in coastal reclamation: A feasibility study. Sugar Cane International Jan/Feb:9-13.

Chimenez, T.A., M.H. Gehlen, K. Marabezi y A.A.S. Curvelo. 2014. Characterization of sugarcane bagasse by autofluorescence microscopy. Cellulose 21:653-664 DOI 10.1007/ s10570-013-0135-9.

Cao Y., S. Shibata y I. Fukumoto. 2006. Mechanical properties of biodegradable composites reinforced with bagasse fibre before and after alkali treatments. Composites: Part A $37: 423-429$.

Clark, J.D.A. 1969. Fibrillation, free water, and fiber bonding. Tappi Journal 52(2):335-340.

COVECA (Comisión Veracruzana de la caña de azúcar). 2008. Monografía de la caña de azúcar. Gobierno del Estado de 
Veracruz. [En línea] http://portal.veracruz.gob.mx/pls/ portal/docs/page/covecainicio/imagenes/archivospdf/ archivosdifusion/monografia\%20ca\%d1adeazucar2010. Pdf. Fecha de acceso: 20/02/2015.

Dasgupta, D., S.K. Suman, D. Pandey, D. Ghosh, R. Khan, D. Agrawal, R.K. Jain, V. T. Vadde y D.K. Adhikari. 2013. Design and optimization of ethanol production from bagasse pith hydrolysate by a thermotolerant yeast $\mathrm{Klu}$ yveromyces sp. IIPE453 using response surface methodology. Springerplus 2:159.

De la Madrid C., E. 2009. La situación de la industria de la celulosa y el papel en el mundo. Financiera Rural. México. [En línea]

http://www.fnd.gob.mx/informacionsectorrural/Documents/ Articulos\%20FR/Microsoft \% 20Word\%20-\%20 art\%C3\%ADculo\%20Celulosa\%20y\%20Papel.pdf. Fecha de acceso: 18/02/2015

Diez, O.A., G.J. Cárdenas y L.F. Mentz. 2010. Poder calorífico superior de bagazo, médula y sus mezclas, provenientes de la caña de azúcar de Tucumán, R. Argentina. Revista industrial y agrícola de Tucumán 87(1):29-38. ISSN 1851-3018.

DOF (Diario Oficial de la Federación). 2014. Programa Nacional de la Agroindustria de la Caña de Azúcar 2014-2018. Secretaría de Gobernación. México. [En línea]

http://dof.gob.mx/nota_detalle.php?codigo $=5343244 \&$ fe cha $=02 / 05 / 2014$. Fecha de acceso: $15 / 02 / 2015$

Emsley, A.M. 2008. Cellulosic ethanol re-ignites the fire of cellulose degradation. Cellulose 15:187-192.

Escoto G., T. 2009. Manual maestro para la evaluación de pulpas a través de la curva de refinación. Universidad de Guadalajara. Ediciones de la noche, Guadalajara, Jalisco, México. 93 p.

FAO (Organización de las Naciones Unidas para la Alimentación y la Agricultura). 2008. Anuario de productos forestales, periodo 2002-2006. Estadística No. 195. Roma. $331 \mathrm{p}$.

Fengel, D. y G. Weneger. 1984. Wood: chemistry, ultrastructure, reactions. Walter de Gruyter. Berlin. 626 p.

Flores, R., R. Muñoz-Ledo, B. Flores y K.I. Cano. 2008. Estimación de la generación de energía a partir de biomasa para proyectos del programa de mecanismo de desarrollo limpio. Revista Mexicana de Ingeniería Química $7(1): 35-39$.

Franklin, G. 1937. Permanent preparations of macerated wood fibres. Tropical Woods 49:21-22.

Freitas Andrade, M. y J.L. Colodette. 2014. Dissolving pulp production from sugar cane bagasse. Industrial Crops and Products 52:58-64.

Garcia-Kirchner, O. y C. Huitron. 1996. Saccharification of native sugar cane bagasse pith by the cross-synergistic action of cellulases from penicillium sp. CH-M-001 and A. terreus CH-M-O 13. Applied Biochemistry and Biotechnology 57/58:253-265.

Ghose, T.K. 1987. Measurement of cellulose activities. Pure and Applied Chemistry 59(2):257-268.

Gilfillan, W., L. Moghaddam y W.O.S. Doherty. 2014. Preparation and characterization of composites from starch with sugarcane bagasse nanofibre. Cellulose 21(4):39 p. DOI 10.1007/s10570-014-0277-4.

González, I., S. Boufi, M.A. Pélach, M. Alcalá, F. Vilaseca y P. Mutjé. 2012. Nanofibrillated cellulose as paper additive in eucaliptus pulps. BioResources 7(4):5167-5180.

Gorres, J., R. Amiri, J.R. Wood y A. Karnis.1996. Mechanical pulp fines and sheet structure. Journal of Pulp and Paper Science 22(12):J490-J496.

Hegbom, L. 1992. Structural aspects of sugar-cane bagasse from a paper-making point of view a light microscopic study. In: Proc. $2^{\text {nd }}$ Int. Non-wood fiber pulping and papermaking conf., Int. Trade Centre, Shanghai. p:657-672.

Hernández-Salas, J.M., M.S. Villa-Ramírez, J.S. Veloz-Rendón, K.N. Rivera-Hernández, R.A. González-César, M.A. Plascencia-Espinosa y S.R. Trejo-Estrada. 2009. Comparative hydrolysis and fermentation of sugarcane and agave bagasse. Bioresource Technology 100:1238-1245.

Howard, R.C. y W. Bichard. 1992. The basic effects of on pulp properties recycling. Journal of Pulp and Paper Science 18(4):151-159.

Htun, M. y A. De Ruvo.1978. The implications of the fines fraction for the properties of bleached kraft sheet. Svensk Papperstidning 81(16):507-510. 
Isaac, A., F. Sketc, C. Driemeierb, G.J.M. Rocha. 2013. 3D imaging of sugarcane bagasse using X-ray microtomography Industrial. Crops and Products 49:790-793.

ISO (International Organization for Standardization).1979. ISO 5264/3. Pulps-laboratory beating- part 3: Jokro mill method. 1979.

ISO (International Organization for Standardization).1999. ISO 5267-1. Pulps-Determination of drainability-Part 1: Schopper-Riegler method.

Jain, R.K., V.V. Thakur, D. Pandey, D.K. Adhikari, A.K. Dixit y R.M. Mathur. 2011. Bioethanol from bagasse pith a lignocellulosic waste biomass from paper/sugar industry. Indian Pulp and Paper Technical Association 23:169-173.

Jayme G. 1944. Mikro-Quellungsmessungen an Zellstoffen. Wochenblatt für Papierfabrikation 6:187-194.

Kordsachia, O., S. Roßkopf y R. Patt. 2004. Production of spruce dissolving pulp with the prehydrolysis-alkaline sulfite process (ph-asa). Lenzinger Berichte 83:24-34.

Lee, S.C. y M. Mariatti. 2008. The effect of bagasse fibers obtained (from rind and pith component) on the properties of unsaturated polyester composites. Materials Letters 62:2253-2256.

Miller, G.L. 1959. Use of dinitrosalicylic acid reagent for determination of reducing sugars. Analytical Chemistry 31(3):426-428.

Minor, J.L., C.T. Scott y H. Atalla. 1993. Restoring bonding strength to recycled fibers. Proceedings recycling symposium. Tappi Press. Atlanta, GA. p:379-385.

Misman M., S.R. Wan Alwi y Z.A. Manan. 2008. State-of-theart for paper recycling. International Conference on Science and Technology (ICSTIE) 12-13.Universiti Teknologi MARA, Pulau Pinang, Malasia.

Nakagaito, A.N. y H. Yano. 2005. Novel high-strength biocomposites based on microfibrillated cellulose having nanoorder-unit web-like network structure. Applied Physics A: Materials Science \& Processing 80(1):55-159.

Novaes, R.C., F.M. Teixeira, C.C. Perrone, C. Sant'Anna, W. de Souza, Y. Abud, E.P. da Silva Bon y V. Ferreira-Leitao. 2012. Structural evaluation of sugar cane bagasse steam pretreated in the presence of $\mathrm{CO}_{2}$ and $\mathrm{SO}_{2}$. Biotechnology for Biofuels 5(36):1-8.
Paavilainen, L. 1993. Conformability-flexibility and collapsibility-of sulphate pulp fibres. Paperi Ja Pun 75(9-10):689702.

Page, D.H. 1969. A theory for the tensile strength of paper. Tappi Journal 52(4):674-681.

Pandey, A., C.R. Soccol, P. Nigam y V.T. Soccol. 2000. Biotechnological potential of agro-industrial residues. I: sugarcane bagasse. Bioresource Technology 74:69-80.

Rainey, T.J., I.M. O’Hara, A.P. Mann, C.H.Bakir y F. Plaza. 2013. Effect of depithing on the safety and ennvironmental aspects of bagasse stockpiling. Process Safety and Environmental Protection 91:378-385.

Rasul, M.G., V. Rudolph y M. Carsky. 1999. Physical properties of bagasse. Fuel 78:905-910.

Rivera J.J., J. Anzaldo, B. Becerra, J. Ramos, R. Sanjuán y J.L. Colodette. 2008. Procesos de blanqueo a base de oxigeno aplicados a pulpa obtenida del cartón corrugado reciclado (OCC). V Congreso Iberoamericano de Investigación en Celulosa y Papel, Ciadicyp. Guadalajara, Jalisco, México.

Rutiaga Quiñones, J.G., J. Anzaldo Hernández, J.J. Vargas Radillo y R. Sanjuán Dueñas. 2002a. Propiedades de resistencia de una pulpa kraft de pino mezclada con médula del bagazo de caña de azúcar. Madera y Bosques $8(2): 17-26$.

Rutiaga Quiñones, J.G., J.J. Rivera Prado y R. Sanjuán Dueñas. 2002b. Blanqueo del parénquima de la caña de azúcar mediante la secuencia $\mathrm{OD}_{1} \mathrm{E}_{\mathrm{p}} \mathrm{D}_{2}$. Ciencia Nicolaita 13:139-145.

Ruzin, S.E. 1999. Plant microtechnique and microscopy. Oxford University Press. Oxford, Nueva York. 322 p.

Sanjuán, R., J.Anzaldo, J. Vargas, J. Turrado y R. Patt. 2001. Morphological and chemical composition of pith and fibers from Mexican sugarcane bagasse. Holz als Rohund Werkstoff 59:447-450.

Scallan, A.M. y A.C. Tydeman. 1992. Swelling and elasticity of the cell walls of pulp fibres. Journal of Pulp and Paper Science 18(5):J188-J193.

TAPPI Test Methods 2002-2003, Tappi Press, Atlanta, GA, USA.

Teixeira, E.D.M., T.J. Bondancia, K.B.R. Teodoro, A.C. Correa, J.M. Marconcini y L.H.C. Mattoso. 2011. Sugarcane 
bagasse whiskers: extraction and characterizations. Industrial Crops and Products 33(1):63-66.

Triana, O., M. Leonard, F. Saavedra, N. Fernández, G. Gálvez y E. Peña. 1990. Atlas del Bagazo de la Caña de Azúcar. Cuba-9. Ed. GEPLACEA/PNUD/ICIDCA, México, D.F. 143 p.

Wagberg, L. 2005. Wood material science. Finnish-Swedish research programme, 2003-2007 year book.

Zheng, Y., Z. Pan y R. Zhang. 2009. Overview of biomass pretreatment for cellulosic ethanol production. International Journal of Agriculture and Biology 2(3):51-68.
Manuscrito recibido el 16 de agosto de 2014. Aceptado el 27 de febrero de 2015.

Este documento se debe citar como:

Vargas-Radillo, J. J., E. Salazar-Ríos, L. Barrientos-Ramírez, A. PérezCenteno, M. Rentería-Urquiza, A. Rodríguez-Rivas, F. Navarro-Arzate y J. Rutiaga-Quiñones. 2015. Pasta blanqueada y azúcares fermentables a partir de médula de bagazo de caña. Madera y Bosques 21(2): 117-130. 